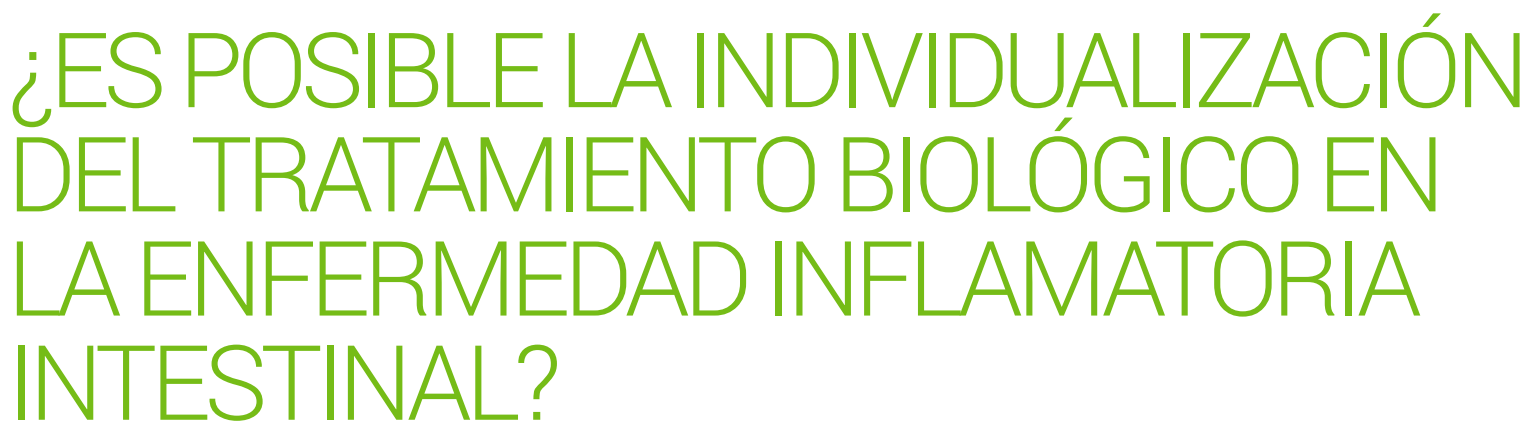

\title{
Is it possible to individualize biological treatment in inflammatory bowel disease?
}

Olmedo Martín RV

HOSPITAL REGIONAL UNIVERSITARIO. MÁLAGA.

\section{Resumen}

La enfermedad inflamatoria intestinal (EII) engloba una serie de trastornos crónicos que afectan primariamente al tracto gastrointestinal, con una patogenia compleja, una presentación clínica heterogénea y una gran variedad de vías inflamatorias que perpetúan el daño intestinal. Tras la introducción de las primeras terapias biológicas, el número de tratamientos se ha ido expandiendo constantemente y se esperan nuevas moléculas en los próximos años. Datos de ensayos clínicos y práctica real, señalan que hasta un $40 \%$ de los pacientes no responden a un tratamiento determinado. Hasta el momento, los clínicos no disponen de herramientas validadas que puedan predecir la respuesta al tratamiento en cada paciente y que guíen en la elección sobre cuál tratamiento administrar. Varios biomarcadores candidatos se han investigado con resultados discordantes: clínicos, genéticos, inmunológicos, farmacocinéticos y microbianos. Recientes datos apoyan que los modelos multiparamétricos parecen prometedores en la predicción de la respuesta al tratamiento. En esta revisión narrativa, nos proponemos resumir el estado actual sobre los predictores y marcadores precoces de respuesta a las terapias biológicas en Ell y la individualización

\section{CORRESPONDENCIA}

Raúl Vicente Olmedo-Martín / raulolmedo1976@yahoo.es Hospital Regional Universitario de Málaga - 29012 Málaga Fecha de envío: 16/11/2021 - Fecha de aceptación: 17/02/2022 del tratamiento, así como dar a conocer las iniciativas en marcha para su desarrollo y aplicación en práctica clínica real.

Palabras clave: enfermedad inflamatoria intestinal, medicina de precisión, tratamiento biológico.

\section{Abstract}

Inflammatory bowel disease (IBD) encompasses a series of chronic disorders that primarily affect the gastrointestinal tract, with a complex pathogenesis, a heterogeneous clinical presentation, and a wide variety of inflammatory pathways that perpetuate intestinal damage. Following the introduction of the first biological therapies, the number of treatments has been constantly expanding and several new molecules are expected in the coming years. Data from clinical trials and real practice indicate that up to $40 \%$ of patients do not respond to a given treatment. Until now, clinicians do not have validated tools that can predict the response to treatment in each patient and that guide the choice of which treatment to administer. Several candidate biomarkers have been investigated with discordant results: clinical, genetic, immunological, pharmacokinetic, and microbial. Recent data support that multiparametric models appear promising in predicting response to treatment. In this narrative review, we intend to summarize the current state of early predictors and markers of response to biological therapies in IBD and the individualization of treatment, as well as to present the 
initiatives underway for their development and application in real clinical practice.

Keywords: inflammatory bowel disease, precision medicine, biologic treatment.

\section{Introducción}

La enfermedad inflamatoria intestinal (EII) engloba a una serie de trastornos inmunomediados que afectan al tracto digestivo, cuyos máximos exponentes son la enfermedad de Crohn (EC) y la colitis ulcerosa. De incidencia y prevalencia crecientes, estas entidades suponen un menoscabo importante en la calidad de vida de los pacientes y un gran impacto sociosanitario ${ }^{1}$. La etiopatogenia de la Ell es compleja y sólo parcialmente dilucidada, interactuando factores ambientales, una predisposición genética del individuo, la microbiota intestinal y alteraciones en la respuesta inmune innata y adaptativa. Distintas vías patogénicas pueden perpetuar el daño intestinal en la Ell, de forma que dos pacientes con un fenotipo similar pueden tener diferentes vías activadas y por tanto, una respuesta distinta a un mismo tratamiento ${ }^{2,3}$. También hay evidencias de que en el mismo paciente, el sistema inmune puede exhibir una importante plasticidad y cambios en la vía inflamatoria predominante en distintos momentos evolutivos de la Ell ${ }^{4}$. Esta complejidad ilustra por qué los tratamientos actuales presentan una eficacia limitada. Mientras el arsenal terapéutico se expande de forma constante, con moléculas dirigidas hacia varias dianas, hay un porcentaje significativo de pacientes que no responden a los tratamientos (datos procedentes de ensayos clínicos y práctica clínica real reportan porcentajes de eficacia no superiores al $60 \%)^{5}$. Además, en el caso de respuesta a un primer agente, en un porcentaje sustancial de pacientes se asistirá a una pérdida de eficacia (fallo secundario) ${ }^{6}$. Teniendo en cuenta este escenario, se antoja necesario el desarrollo de nuevas herramientas o biomarcadores que puedan predecir en primer lugar qué pacientes se beneficiarían de la introducción precoz de terapias avanzadas, posteriormente la probabilidad de respuesta del paciente a un agente concreto y por último evaluar de forma temprana si el paciente responde. La implementación de la medicina personalizada en el manejo rutinario de la Ell va a ser el mayor desafío en las próximas décadas, y va a tener como objetivo el proporcionar el mejor cuidado a los pacientes con una reducción paralela de los costes para los sistemas de salud. En esta revisión narrativa nos proponemos revisar el conocimiento actual sobre predictores y biomarcadores de respuesta precoz al tratamiento biológico de la ElI.

\section{Factores predictivos y/o biomarcadores tradicionales}

La asociación de las características del paciente y la enfermedad con la respuesta al tratamiento ha sido muy investigada, pero los resultados son discordantes. Edad, sexo, peso y tabaquismo no han mostrado una correlación unánime con la respuesta al tratamiento anti-TNFa ni otros agentes ${ }^{7}$. Dos recientes metaanálisis sugieren que el tratamiento precoz en EC se asocia a mejores porcentajes de respuesta y curación mucosa, aunque sin diferencias entre distintas clases de tratamiento 8,9 . En cuanto a la localización de la Ell, en la EC no parece influir en una mejor respuesta al tratamiento con infliximab o ustekinumab. En el caso de vedolizumab, mientras un estudio le asignaba mejor probabilidad de respuesta a la localización colónica, esto no ha sido confirmado por otros. Además si bien algunos trabajos sugieren una mejor respuesta a antiTNFa del fenotipo inflamatorio, ello no se ha visto corroborado para vedolizumab o ustekinumab ${ }^{10,11}$. En cuanto a los marcadores serológicos, un reciente estudio retrospectivo en pacientes mayores de 60 años encontró que una mayor ratio de hormonas tiroideas T3/T4 se asoció a cicatrización mucosa independendientemente del agente utilizado ${ }^{12}$. Calprotectina fecal (CF) y proteína $\mathrm{C}$ reactiva han mostrado también resultados discordantes y conflictivos en cuanto a la predicción de la respuesta. En este sentido, un estudio demostró una asociación entre unos niveles bajos de CF postinducción con una mayor probabilidad de respuesta sostenida y curación mucosa tras el tratamiento antiTNF ${ }^{13}$. La exposición previa a un agente biológico y la razón de la suspensión del primer biológico (mayor posibilidad de respuesta si intolerancia previa a antiTNF versus fallo primario o secundario) también han sido propuestos. Por último se ha sugerido la pertinencia de cambio de mecanismo de acción frente al cambio por otro fármaco de la misma clase en caso de fallo primario.

\section{Marcadores genéticos}

Los estudios genéticos han contribuido de forma importante al mejor conocimiento de la fisiopatología de la Ell y al desarrollo de nuevas dianas terapéuticas. No obstante, los marcadores genéticos generalmente muestran un rendimiento bajo en la predicción de respuesta a un determinado agente. En la tabla 1 resumimos los estudios que han investigado la relación entre los marcadores genéticos y la respuesta al tratamiento biológico ${ }^{14}$

De todos ellos, son de especial interés los capaces de predecir la generación de anticuerpos contra infliximab y adalimumab, ya que este aspecto es fundamental en la pérdida de respuesta al fármaco. En este sentido, la cohorte PANTS, mediante estudio de genoma completo, mostró que la presencia de una o más copias del alelo HLA-DQA 1 *05 confería casi el doble de riesgo de desarrollar inmunogenicidad ${ }^{15}$. Por ello en pacientes con la presencia de 1 o más copias de este alelo la comboterapia sería aconsejable para evitar la pérdida de respuesta secundaria. El ensayo clínico INHERIT [NCT04109300] pretende evaluar la utilidad clínica de solicitar el HLA-DQA $1 * 05$ antes del inicio de infliximab en una cohorte canadiense. En relación a marcadores genéticos asociados a la respuesta a anti-integrinas, sólo la expresión del gen aE fue predictivo de remisión clínica en la semana 10 en la fase 2 de un ensayo clínico con etrolizumab en $\mathrm{CU}^{16}$. No se han descrito marcadores genéticos asociados con la respuesta a agentes antilL-12/23.

\section{Otros biomarcadores: transcriptómica, proteómica y marcadores inmunológicos}

Los estudios de transcriptómica han ofrecido resultados más esperanzadores, sugiriendo que la respuesta a los diferentes biológicos parece depender de la expresión 


\begin{tabular}{|c|c|}
\hline Marcador genético & $\begin{array}{l}\text { Asociación con la respuesta } \\
\text { al tratamiento biológico }\end{array}$ \\
\hline $\begin{array}{l}\text { Polimorfismos en TLR2, rs11938228, } \\
\text { TLR4, TLR9, TNFRSF1A, IFNG, IL6 } \\
\text { and IL1B (rs4848306) }{ }^{14}\end{array}$ & Respuesta clínica a antiTNFa en Ell \\
\hline Polimorfismos en IL23R ${ }^{16}$ & Respuesta precoz a infliximab en CU \\
\hline HLA-DRB1 $1^{24}$ & $\begin{array}{l}\text { Desarrollo de anticuerpos anti-infliximab } \\
\text { en Ell }\end{array}$ \\
\hline HLA-DQA $1 * 05^{15}$ & $\begin{array}{l}\text { Desarrollo de anticuerpos anti-infliximab y } \\
\text { anti-adalimumab en EC }\end{array}$ \\
\hline $\begin{array}{l}\text { Polimorfismo en el receptor } \\
\text { FGCR3A }\end{array}$ & $\begin{array}{l}\text { Desarrollo de anticuerpos anti-infliximab } \\
\text { en EC }\end{array}$ \\
\hline Polimorfismos en NOD2 ${ }^{16}$ & Niveles de antiTNF más bajos en EC \\
\hline Polimorfismos en ATG16L1 $1^{16}$ & Respuesta clínica a adalimumab en $\mathrm{EC}$ \\
\hline $\begin{array}{l}\text { Polimorfismos } \\
\text { TNFSF4/18, PLIN2, rs762787, } \\
\text { rs } 9572250, \text { rs } 144256942, \\
\text { rs523781 }\end{array}$ & Respuesta clínica a antiTNF en Ell \\
\hline
\end{tabular}

\section{TABLA 1}

MARCADORES GENÉTICOS Y ASOCIACIÓN CON LA RESPUESTA AL

diferencial de genes, más que de variantes genéticas. Uno de los biomarcadores más prometedores es el TREM-1 (triggering receptor expressed on myeloid cells 1) cuya reducción en sangre completa y en mucosa intestinal se asoció con curación mucosa tras tratamiento con antiTNFa, pero no al evaluarlo en pacientes con vedolizumab ${ }^{17}$. Una alta expresión de un miembro de la familia de la IL-6, oncostatin $M$, se ha relacionado con la refractariedad a antiTNF tanto en pacientes con EC como $\mathrm{CU}^{18}$. El incremento sérico de VCAM-1 e ICAM-1 y el descenso de a4ß3 se asociaron a remisión clínica y endoscópica en pacientes tratados con vedolizumab ${ }^{19}$. En el ensayo fase 2 de brazikumab (un anti-IL23), las concentraciones basales elevadas de IL-22 se asoció con la probabilidad de respuesta al tratamiento ${ }^{20}$. Los resultados de los principales estudios de transcriptómica y estudios inmunológicos se resumen en la tabla 2.

\section{Monitorización de niveles}

La monitorización de niveles de biológicos consiste en la determinación de niveles valle del fármaco y de la presencia de anticuerpos contra el mismo. En el momento actual, se recomienda la monitorización reactiva de niveles (pacientes con actividad clínica) en el caso de pérdida de respuesta a agentes antiTNFa, principalmente infliximab o adalimumab ${ }^{21,22}$. Además esta estrategia se ha demostrado más costo-efectiva en el manejo de la pérdida de respuesta secundaria que la

\begin{tabular}{|c|c|}
\hline Marcadores inmunológicos & $\begin{array}{l}\text { Asociación con la respuesta } \\
\text { al tratamiento biológico }\end{array}$ \\
\hline $\begin{array}{l}\text { Baja expresión de TREM-1 en sangre } \\
\text { total y biopsias intestinales, baja } \\
\text { concentración en suero }{ }^{17}\end{array}$ & $\begin{array}{l}\text { Curación mucosa en pacientes tratados } \\
\text { con antiTNF }\end{array}$ \\
\hline $\begin{array}{l}\text { Elevado numero de células mTNF+ } \\
\text { en biopsias intestinales }{ }^{17}\end{array}$ & $\begin{array}{l}\text { Respuesta a corto plazo (12 semanas) a } \\
\text { adalimumab }\end{array}$ \\
\hline $\begin{array}{l}\text { Alta expresión de oncostatin } M \text { en } \\
\text { biopsias intestinales }{ }^{18}\end{array}$ & $\begin{array}{l}\text { Refractariedad a golimumab (semana 6) e } \\
\text { infliximab (semanas } 8 \text { y } 30 \text { ) }\end{array}$ \\
\hline $\begin{array}{l}\text { Concentración sérica baja de } \\
\text { oncostatin } \mathrm{M}^{18}\end{array}$ & $\begin{array}{l}\text { Curación mucosa en semana } 54 \text { en } \\
\text { pacients tratados con infliximab }\end{array}$ \\
\hline $\begin{array}{l}\text { VCAM-1 e ICAM-1 elevados en } \\
\text { suero y bajo nivel séricos de } a 4 \beta 7^{19}\end{array}$ & $\begin{array}{l}\text { Remisión endoscópica en pacientes } \\
\text { tratados con vedolizumab }\end{array}$ \\
\hline Niveles séricos altos de IL-22 20 & Respuesta clínica a brazikumab (anti-IL23) \\
\hline
\end{tabular}

TABLA 2

MARCADORES INMUNOLÓGICOS Y DE TRANSCRIPTÓMICA Y SU ASOCIACIÓN CON LA RESPUESTA AL TRATAMIENTO.

escalada de dosis basada meramente en los síntomas ${ }^{23,24}$. Recientemente el estudio PANTS donde se incluyeron al tiempo del inicio del tratamiento pacientes antiTNF naive con EC luminal, y fueron seguidos hasta la suspensión del tratamiento, reveló que niveles de IFX ${ }^{25}$.

En un trabajo de 2018, Roblin et al observaron que el estado farmacocinético en el momento del fallo al primer antiTNF, podía ayudar a predecir los resultados de un segundo antiTNF: los pacientes con niveles adecuados en el momento de la pérdida de eficacia tuvieron significativamente menos probabilidades de conseguir remisión en la semana 30 con respecto a pacientes con niveles infraterapéuticos o indetectables independientemente de la titulación de anticuerpos contra el fármaco. La probabilidad de desarrollar anticuerpos antifármaco con un segundo antiTNF fue también mayor en el caso de niveles indetectables y anticuerpos positivos. Este mismo grupo, en línea con lo anterior, reportó que la adición de un inmunosupresor a un segundo antiTNF en caso de fallo previo por inmunogenicidad puede reducir la formación de anticuerpos y mejorar los resultados ${ }^{26,27}$.

Si bien no hay evidencias para recomendar la determinación proactiva de niveles (TAXIT y TAILORIX), hay datos emergentes sobre la asociación entre niveles de fármaco en fase de inducción y la eficacia del tratamiento, sugiriendo una potencial herramienta de prevención del fallo primario ${ }^{28,29}$.

Los datos referentes a vedolizumab o ustekinumab son escasos. Son agentes que exhiben una menor inmunogenicidad comparado a los antiTNF, y la presencia de anticuerpos contra ambos no se ha asociado de forma consistente al fracaso 
terapeútico en los ensayos clínicos pivotales. Debido a la heterogeneidad de test usados para medir niveles, diferentes momentos elegidos y variables pronósticas investigadas, los puntos de corte de niveles de vedolizumab propuestos son variables. Una revisión reciente propuso niveles objetivo para vedolizumab y ustekinumab. En la inducción, niveles de vedolizumab de $>20 \mathrm{mcg} / \mathrm{mL}$ en semana 6 y $>14 \mathrm{mcg} / \mathrm{ml}$ en semana 14, mientras que para ustekinumab serían de $>14$ $\mathrm{mcg} / \mathrm{mL}$ y $>4 \mathrm{mcg} / \mathrm{mL}$ en semanas 4 y 8 respectivamente. En el mantenimiento proponen concentraciones de vedolizumab $>12$ $\mathrm{mcg} / \mathrm{mL}$ y $>2,5 \mathrm{mcg} / \mathrm{mL}$ para ustekinumab ${ }^{30}$.

En la tabla 3 se recogen algunos de los principales estudios de monitorización de niveles que podrían facilitar una individualización del tratamiento biológico.

\begin{tabular}{|c|c|}
\hline Puntos de corte & $\begin{array}{l}\text { Asociación con la respuesta } \\
\text { al tratamiento biológico }\end{array}$ \\
\hline $\begin{array}{l}\text { Infliximab } \geq 7,5 \mu \mathrm{g} / \mathrm{mL} \quad \text { en } \quad \text { el } \\
\text { mantenimiento21 }\end{array}$ & $\begin{array}{l}\text { Curación mucosa en pacientes tratados } \\
\text { con antiTNF en EC }\end{array}$ \\
\hline $\begin{array}{l}\text { Infliximab }>7 \mu \mathrm{g} / \mathrm{mL} \text { en semana } 14 \\
\text { Adalimumab }>12 \mu \mathrm{g} / \mathrm{mL} \text { en semana } \\
1422\end{array}$ & $\begin{array}{l}\text { Remisión clínica en semana } 14 \text { y } 54 \text { en } \\
\text { EC }\end{array}$ \\
\hline $\begin{array}{l}\text { Vedolizumab } \geq 20,9 \mu \mathrm{g} / \mathrm{mL} \text { en semana } \\
6 y \geq 10.1 \text { en semana } 1423.24\end{array}$ & $\begin{array}{l}\text { Mejoría endoscópica en semana } 14 \\
\text { Remisión endoscópica a los } 6 \text { meses }\end{array}$ \\
\hline $\begin{array}{l}\text { Vedolizumab } \geq 27 \mu \mathrm{g} / \mathrm{mL} \text { durante el } \\
\text { mantenimiento30 }\end{array}$ & Remisión clínica en semana 52 (EC y CU) \\
\hline $\begin{array}{l}\text { Vedolizumab } 7,4 \mu \mathrm{g} / \mathrm{mL} \text { antes de la } \\
\text { escalada de dosis } 30\end{array}$ & $\begin{array}{l}\text { Respuesta a escalada de dosis en } \\
\text { pacientes tratados con vedolizumab }\end{array}$ \\
\hline $\begin{array}{l}\text { Ustekinumab } \geq 3,3 \mu \mathrm{g} / \mathrm{mL} \text { en semana } 8 \\
\text { Ustekinumab } 0,8-1,4 \mu \mathrm{g} / \mathrm{mL} \text { durante } \\
\text { el mantenimiento } 30\end{array}$ & $\begin{array}{l}\text { Remisión clínica en semana } 8 \text { (EC) } \\
\text { Remisión clínica durante el } \\
\text { mantenimiento }(E C)\end{array}$ \\
\hline $\begin{array}{l}\text { Ustekinumab } \geq 3,7 \mu \mathrm{g} / \mathrm{mL} \text { en semana } 8 \\
\text { Ustekinumab } 1,1-1,3 \mu \mathrm{g} / \mathrm{mL} \text { durante } \\
\text { el mantenimiento30 }\end{array}$ & $\begin{array}{l}\text { Remisión clínica semana } 8 \text { (CU) } \\
\text { Remisión clínica semana } 44 \text { (CU) }\end{array}$ \\
\hline
\end{tabular}

TABLA 3

PRINCIPALES ESTUDIOS DE MONITORIZACIÓN DE NIVELES QUE PODRÍAN FACILITAR UNA INDIVIDUALIZACIÓN DEL TRATAMIENTO BIOLÓGICO.

\section{Microbiota intestinal}

Mientras está bien establecido que la microbiota intestinal desempeña un papel clave en la patogénesis de la Ell, si la disbiosis es una causa o una consecuencia de inflamación intestinal no está aclarado. Además, junto a las alteraciones en la composición de la microbiota, cambios en las funciones metabólicas de la microbiota pueden tener un impacto en la fisiopatología de la Ell ${ }^{31}$. La tabla 4 recoge algunas de las principales asociaciones entre diversas características de la microbiota y la respuesta al tratamiento ${ }^{32}$.

\section{Iniciativas en marcha mediante aproximaciones multiómicas}

La aplicación de la multiómica, que incorpora algunos de los elementos que hemos reseñado en apartados anteriores pero de forma conjunta, ha mostrado resultados prometedores.

\begin{tabular}{|c|c|}
\hline Marcadores microbianos & $\begin{array}{l}\text { Asociación con la respuesta al tratamiento } \\
\text { biológico }\end{array}$ \\
\hline $\begin{array}{l}\text { Menor índice de disbiosis y } \\
\text { abundancia de F. prausnitizii } 31\end{array}$ & Respuesta clínica a anti-TNF en Ell \\
\hline $\begin{array}{l}\text { Abundancia de bacterias productoras } \\
\text { de ácidos grasos de cadena corta31 }\end{array}$ & Repuesta sostenida a IFX en EC \\
\hline $\begin{array}{l}\text { Alta diversidad y riqueza bacteriana } \\
\text { y abundancia relativa de } \mathrm{F} \text {. } \\
\text { prausnitzii31 }\end{array}$ & $\begin{array}{l}\text { Infliximab }>5 \mu \mathrm{g} / \mathrm{mL} \text { en semana } 8 \text { y } \\
\text { curación mucosa a los } 3 \text { meses en Ell }\end{array}$ \\
\hline $\begin{array}{l}\text { Mayor diversidad y abundancia } \\
\text { de Roseburia inulinivorans y de } \\
\text { Burkholderiales } 32\end{array}$ & $\begin{array}{l}\text { Remisión clínica en pacientes tratados con } \\
\text { vedolizumab }\end{array}$ \\
\hline $\begin{array}{l}\text { Vías metabólicas asociadas con } \\
\text { funciones microbianas } 32\end{array}$ & \\
\hline $\begin{array}{l}\text { Mayor diversidad y abundancia de } \\
\text { unidades taxonómicas operacionales } \\
\text { afiliadas con Faecalibacterium y } \\
\text { Bacteroides32 }\end{array}$ & $\begin{array}{l}\text { Remisión clínica a las } 6 \text { semanas en EC } \\
\text { tratada con ustekinumab }\end{array}$ \\
\hline
\end{tabular}

TABLA 4

PRINCIPALES ASOCIACIONES ENTRE DIVERSAS CARACTERÍSTICAS DE LA MICROBIOTA Y LA RESPUESTA AL TRATAMIENTO.

Otros trabajos están en desarrollo para validación de estos datos en cohortes independientes. Idealmente estas iniciativas deberían ser apoyadas por otros estudios de multiómica de grandes cohortes colaborativas como el IBD BioResource en Reino Unido y el proyecto IBD Multiomics Data en Estados Unidos $^{33}$. Estas aproximaciones utilizando big data, biología de sistemas y técnicas de machine learning, entre otras, deben facilitar el descubrimiento y la implementación de biomarcadores que hagan la medicina personalizada una realidad en la El|34.

\section{Conclusiones}

El paradigma "one size fits all" donde no existe una adaptación personalizada del tratamiento médico ha sido rechazada desde el punto de vista conceptual en la Ell. Sin embargo, estamos lejos de disponer de herramientas pronósticas válidas que puedan ser aplicadas en práctica clínica. Los marcadores del paciente y la enfermedad tienen un papel en predecir el curso y agresividad de la Ell en un determinado paciente, pero no poseen la capacidad adecuada para discernir quién responderá a un determinado tratamiento. Los marcadores genéticos y transcriptómicos tienen el potencial de predecir la respuesta a los tratamientos de la Ell; se han investigado un nutrido número de loci pero ninguno se ha mostrado ideal. La susceptibilidad a la formación de anticuerpos antifármaco conferida por algunos polimorfismos genéticos, podría ser de gran utilidad en práctica real en la identificación de pacientes que podrían beneficiarse 
de la asociación de inmunosupresores. Con respecto a la determinación de niveles, se desconoce todavía si los niveles terapéuticos más altos se relacionan causalmente con mejores resultados o son sólo un marcador de la carga inflamatoria y la eficacia del tratamiento. El paisaje inmunológico de la Ell se caracteriza por una variabilidad y plasticidad notable inter e intra-paciente; de forma que, una mayor comprensión de las vías inflamatorias predominantes activadas en un paciente en un tiempo específico podría ayudar sustancialmente a guiar el tratamiento. Por último, los marcadores microbiológicos presentan un gran atractivo. La microbiota intestinal parece cambiar en paralelo con las distintas fases de la Ell y podría ser considerado como un marcador no invasivo y dinámico que podría informar de la actividad de la Ell y predecir la respuesta a los tratamientos. De forma interesante, la mayoría de estudios recientes parecen sugerir que los modelos multiparamétricos, incorporando diferentes disciplinas, proporcionan los valores predictivos más altas. Como conclusión, la implementación de la medicina personalizada es una de las necesidades no cubiertas en Ell. Dado el incremento de la prevalencia de la Ell, los costes sociosanitarios derivados y el aumento del arsenal terapéutico, deben diseñarse estudios que busquen el desarrollo de nuevas herramientas pronósticas que nos permitan elegir el tratamiento correcto, para un paciente concreto y en el momento adecuado.

\section{Bibliografía}

1. Kaplan G.G. The global burden of IBD: from 2015 to 2025. Nat. Rev. Gastroenterol. Hepatol. 2015;12: 720-727.

2. VerdierJ, Bègue B, Cerf-Bensussan N, Ruemmele, F.M. Compartmentalized expression of Th1 and Th17 cytokines in pediatric inflammatory bowel diseases. Inflamm. Bowel Dis. 2012; 18:1260-1266.

3. Atreya R, Neurath M.F. Mechanisms of molecular resistance and predictors of response to biological therapy in inflammatory bowel disease. Lancet Gastroenterol. Hepatol. 2018; 3: 790-802.

4. Zorzi F, Monteleone I, Sarra M, Calabrese E, Marafini I, Cretella M, et al. Distinct profiles of effector cytokines mark the different phases of Crohn's disease. PLOS ONE 2013, 8, e54562.

5. Singh S, Murad M.H, Fumery M, Dulai P.S, Sandborn W.J. First- and second-line pharmacotherapies for patients with moderate to severely active ulcerative colitis: an updated network meta-analysis. Clin. Gastroenterol. Hepatol. 2020; 18: 2179-219.

6. Ben-Horin S, Kopylov U, Chowers Y. Optimizing anti-TNF treatments in inflammatory bowel disease. Autoimmun. Rev. 2014;13: 24-30.

7. Gisbert J.P, Chaparro M. Predictors of primary response to biologic treatment [Anti-TNF, Vedolizumab, and Ustekinumab] in patients with Inflammatory Bowel Disease: from basic science to clinical practice. J. Crohn's Colitis. 2020; 14: 694-709.
8. Hamdeh S, Aziz M, Altayar O, Olyaee M, Murad M.H, Hanauer, S.B. Early vs late use of anti-TNFa therapy in adult patients with Crohn Disease: $A$ systematic review and meta-analysis. Inflamm. Bowel Dis. 2020; 26:18081818.

9. Ungaro R.C, Aggarwal S, Topaloglu O, Lee W, Clark R, Colombel, J.-F. Systematic review and meta-analysis: efficacy and safety of early biologic treatment in adult and paediatric patients with Crohn's disease. Aliment. Pharmacol. Ther. 2020; 51:831-842.

10. Zorzi F, Zuzzi S, Onali S, Calabrese E, Condino G, Petruzziello C, et al.. Efficacy and safety of infliximab and adalimumab in Crohn's disease: $A$ single centre study. Aliment. Pharmacol. Ther. 2012; 35:1397-1407.

11. Singh N, Rabizadeh S, Jossen J, Pittman N, Check M, Hashemi G, et al. Multi-center experience of vedolizumab effectiveness in pediatric inflammatory bowel disease. Inflamm. Bowel Dis. 2016; 22: 2121-2126

12. Bertani L, Tricò D, Pugliese D, Privitera G, Linsalata G, Zanzi F, et al. Serum triiodothyronine-to-thyroxine (T3/T4) ratio predicts therapeutic outcome to biological therapies in elderly IBD patients.. Aliment. Pharmacol. Ther. 2020, 53:273-280.

13. Guidi L, Marzo M, Andrisani G, Felice C, Pugliese D, Mocci G, et al.Faecal calprotectin assay after induction with anti-tumour necrosis factor agents in inflammatory bowel disease: Prediction of clinical response and mucosal healing at one year. Dig. Liver Dis. 2014; 46: 974-979.

14. Bek S, Nielsen J.V, Bojesen A.B, Franke A, Bank S, Vogel U,et al. Systematic review: genetic biomarkers associated with anti-TNF treatment response in inflammatory bowel diseases. Aliment. Pharmacol. Ther. 2016; 44: 554-567.

15. Sazonovs A, Kennedy N.A, Moutsianas L, Heap G.A, Rice D.L, Reppell, $M$, et al. HLA-DQA ${ }^{*} 05$ carriage associated with development of anti-drug antibodies to infliximab and adalimumab in patients with Crohn's Disease. Gastroenterology. 2020; 158: 189-199.

16. Vermeire S, O'Byrne S, Keir M, Williams M, Lu T.T, Mansfiel J.C, et al. Etrolizumab as induction therapy for ulcerative colitis: A randomised, controlled, phase 2 trial. Lancet. 2014; 384: 309-318.

17. Verstockt, B.; Verstockt, S.; Dehairs, J.; Ballet, V.; Blevi, H.;Wollants,W.-J, et al. LOW TREM1 expression in whole blood predicts anti-TNF response in inflammatory bowel disease. EBioMedicine 2019; 40: 733-742.

18. West N.R, Hegazy A.N, Owens B.M.J, Bullers S.J, Lingg, B, Buonocore $S$, et al. Oncostatin $M$ drives intestinal inflammation and predicts response to tumor necrosis factor-neutralizing therapy in patients with inflammatory bowel disease. Nat. Med. 2017; 23:579-589

19. Holmer A.K, Battat R, Dulai P.S, Casteele N.V, Nguyen N, Jain A. et al. Biomarkers are associated with clinical and endoscopic outcomes with vedolizumab treatment in Crohn's disease. Ther. Adv. Gastroenterol. 2020, 13.

20. Sands B.E, Chen J, Feagan B.G, Penne M, Rees W.A, Danese S et al. Efficacy and safety of MEDI2070, an antibody against interleukin 23, in patients with moderate to severe Crohn's Disease: a phase 2a study. 
Gastroenterology. 2017; 153:77-86.e6.

21. Mitrev N, Casteele N.V, Seow C.H, Andrews J.M, Connor S.J, Moore G.T, et al. Review article: Consensus statements on therapeutic drug monitoring of anti-tumour necrosis factor therapy in inflammatory bowel diseases. Aliment. Pharmacol. Ther. 2017; 46: 1037-1053.

22. Guidi L, Pugliese D, Tonucci T.P, Berrino A, Tolusso B, Basile M, et al. Therapeutic drug monitoring is more cost-effective than a clinically based approach in the aanagement of loss of esponse to infliximab in inflammatory bowel disease: an observational multicentre study. J. Crohn's Colitis. 2018;12: 1079-1088.

23. Steenholdt C, Brynskov J, Thomsen O, Østergaard M, Munck L.K, Fallingborg J, et al. Individualised therapy is more cost-effective than dose intensification in patients with Crohn's disease who lose response to antiTNF treatment: a randomised, controlled trial. Gut. 2014; 63: 919-927.

24. Argollo M, Kotze P.G, Kakkadasam P, D'Haens, G. Optimizing biologic therapy in IBD: How essential is therapeutic drug monitoring? Nat. Rev. Gastroenterol. Hepatol. 2020; 17: 1-9.

25. Kennedy N.A, Heap G.A, Green H.D, Hamilton B, Bewshea C, Walker, G.J, et al. Predictors of anti-TNF treatment failure in anti-TNF-naive patients with active luminal Crohn's disease: A prospective, multicentre, cohort study. Lancet Gastroenterol. Hepatol. 2019; 4: 341-353.

26. Roblin X, Marotte H, Rinaudo M, Del Tedesco E, Moreau A, Phelip J.M, et al. Association between pharmacokinetics of adalimumab and mucosal healing in patients with inflammatory bowel diseases. Clin. Gastroenterol. Hepatol. 2014; 12: 80-84.

27. Paul S, Del Tedesco E, Marotte H, Rinaudo-Gaujous M, Moreau A, Phelip, J.M, et al.Therapeutic drug monitoring of infliximab and mucosal healing in inflammatory bowel disease. Inflamm. Bowel Dis. 2013; 19: 2568-2576.

28. Casteele N.V, Ferrante M, Van Assche G, Ballet V, Compernolle G, Van Steen $K$, et al. Trough concentrations of infliximab guide dosing for patients with inflammatory bowel disease. Gastroenterology. 2015; 148 : 1320-1329

29. D'Haens, G, Vermeire, S, Lambrecht, G, Baert, F, Bossuyt, P, Pariente, $B$, et al. Increasing infliximab dose based on symptoms, biomarkers, and serum drug concentrations does not increase clinical, endoscopic, and corticosteroid-free remission in patients with active luminal Crohn's Disease. Gastroenterology. 2018;154:1343-1351.

30. Alsoud D, Vermeire S, Verstockt B. Monitoring vedolizumab and ustekinumab drug levels in patients with inflammatory bowel disease: Hype or hope? Curr. Opin. Pharmacol. 2020; 55: 17-30.

31. Lee M, Chang E.B. Inflammatory Bowel Diseases (IBD) and the microbiome-searching the crime scene for clues. Gastroenterology. 202;160: 524-537.

32. Estevinho M.M, Rocha C, Correia L, Lago P, Ministro P, Portela F, et al.Features of fecal and colon microbiomes associate with responses to biologic therapies for inflammatory bowel diseases: a systematic review. Clin. Gastroenterol. Hepatol. 2020; 18: 1054-1069

33. Verstockt B, Noor NM, Marigorta UM, Pavlidis $P$, Deepak $P$, Ungaro RC, et al. Scientific Workshop Steering Committee. Results of the Seventh Scientific Workshop of ECCO: Precision Medicine in IBD-Disease Outcome and Response to Therapy. J Crohns Colitis. 2021 Sep 25;15:1431-1442

34. Olivera P, Danese S, Jay N, Natoli G, Peyrin-Biroulet L. Big data in IBD: a look into the future. Nat Rev Gastroenterol Hepatol. 2019 May;16:312-321 